\title{
Common-Mode Suppression in Microstrip Differential Lines by Means of Complementary Split Ring Resonators: Theory and Applications
}

\author{
Jordi Naqui, Student Member, IEEE, Armando Fernández-Prieto, Student Member, IEEE, \\ Miguel Durán-Sindreu, Member, IEEE, Francisco Mesa, Senior Member, IEEE, Jesús Martel, Member, IEEE, \\ Francisco Medina, Fellow, IEEE, and Ferran Martín, Fellow, IEEE
}

\begin{abstract}
This paper is focused on the application of complementary split-ring resonators (CSRRs) to the suppression of the common (even) mode in microstrip differential transmission lines. By periodically and symmetrically etching CSRRs in the ground plane of microstrip differential lines, the common mode can be efficiently suppressed over a wide band whereas the differential signals are not affected. Throughout the paper, we present and discuss the principle for the selective common-mode suppression, the circuit model of the structure (including the models under even- and odd-mode excitation), the strategies for bandwidth enhancement of the rejected common mode, and a methodology for common-mode filter design. On the basis of the dispersion relation for the common mode, it is shown that the maximum achievable rejection bandwidth can be estimated. Finally, theory is validated by designing and measuring a differential line and a balanced bandpass filter with common-mode suppression, where double-slit CSRRs (DSCSRRs) are used in order to enhance the common-mode rejection bandwidth. Due to the presence of DS-CSRRs, the balanced filter exhibits more than $40 \mathrm{~dB}$ of common-mode rejection within a $34 \%$ bandwidth around the filter pass band.
\end{abstract}

Index Terms-Complementary split-ring resonator (CSRR), differential transmission lines, metamaterial-inspired lines, split-ring resonator (SRR).

\section{INTRODUCTION}

I $\mathrm{N}$ a recent work presented by the authors [1], it was demonstrated that complementary split-ring resonators (CSRRs) [2] are useful for the suppression of the even (common) mode in microstrip differential lines. The suppression of the even mode is of interest in high-speed digital circuits based on differential

Manuscript received March 05, 2012; revised June 25, 2012; accepted June 27, 2012. Date of publication August 28, 2012; date of current version September 27, 2012. This work was supported in part by MICIIN-Spain under Contract TEC2010-17512, Contract TEC2010-16948, and Contract TEC2011-13615-E, Generalitat de Catalunya under Project 2009SGR-421 and VALTEC08-1-0009, and by MICIIN-Spain Project CONSOLIDER EMET CSD2008-00066. The work of J. Naqui was supported by MICIIN-Spain through FPU Grant AP2010-0431.

J. Naqui, M. Durán-Sindreu, and F. Martín are with GEMMA/CIMITEC, Departament d'Enginyeria Electrònica, Universitat Autònoma de Barcelona, 08193 Bellaterra, Spain (e-mail: Ferran.Martin@uab.es).

A. Fernández-Prieto, F. Mesa, J. Martel, and F. Medina are with Grupo de Microondas, Universidad de Sevilla, 41012 Sevilla, Spain (e-mail: Medina@us. es).

Color versions of one or more of the figures in this paper are available online at http://ieeexplore.ieee.org.

Digital Object Identifier 10.1109/TMTT.2012.2209675 signals in order to reduce common-mode radiation and electromagnetic interference (EMI). Prior to our work [1], several approaches were reported to efficiently suppress the even mode while keeping the integrity of the differential signals in differential transmission lines. The common-mode filters based on lowtemperature cofired ceramic (LTCC) technology reported in [3] or the negative permeability structures of [4] are compact and provide efficient common-mode rejection over wide frequency bands, but they are technologically complex. Common-mode filters based on defected ground structures were also reported. In [5], dumbbell-shaped periodic patterns etched in the ground plane, underneath the differential lines, were used to suppress the even mode by opening the return current path through the ground plane. This has a small effect on the differential signals (odd mode), since relatively small current density returns through the ground plane for such signals. In [6], a wide stopband for the common mode was achieved by using U-shaped and $\mathrm{H}$-shaped coupled resonators symmetrically etched in the ground plane. A comparison of the previous approaches, including our CSRR-based common-mode filters, was reported in [1], and it was found that the combination of size, bandwidth, and stopband rejection achievable by means of CSRR-loaded differential lines is very competitive.

As reported in [1], tightly coupled CSRRs are necessary [7] to enhance the rejection bandwidth of the common mode. To gain insight on this, the dispersion relation of the equivalent circuit model for the common mode, including inter-resonator coupling, will be obtained. Due to the electric coupling between adjacent CSRRs, the equivalent circuit model of the unit cell for the common mode consists of a four-port network. By applying Floquet's analysis to the circuit, the dispersion relation will be inferred, and it will be demonstrated that bandwidth enhancement is due to the destructive interference of complex modes (see, for instance, [8] and references therein).

It was also shown in [1] that, to enhance the rejection bandwidth of an individual resonator (common mode), it is necessary to increase the coupling capacitance between the pair of lines and the CSRR and to decrease the inductance and capacitance of the CSRR. This was achieved in [1] by increasing the rings width and separation. Alternatively, it will be shown in this paper that bandwidth enhancement can be obtained by replacing the CSRRs with double-slit CSRRs (DS-CSRRs) [9]. The advantage of using DS-CSRRs is that the bandwidth can be enhanced while preserving the description of the particle 
through an $L C$ resonant tank. The validity of the circuit model is important for synthesis purposes. This means that broadband common-mode filters based on largely separated and wide slot rings can be implemented (as was demonstrated in [1]), but not following a systematic approach, which is one of the main motivations of the present work.

This paper is organized as follows. The principle for selective common-mode suppression in microstrip differential lines loaded with CSRRs is presented in Section II. The equivalent circuit model of the structure, as well as the models that result from even- and odd-mode excitation, are presented and used to validate the principle for common-mode suppression. As long as the proposed common-mode filters can be considered as truncated periodic structures, the knowledge of the dispersion relation of these CSRR-loaded differential lines is a powerful tool to estimating the maximum achievable common mode rejection bandwidth. Thus, Section III is devoted to infer the dispersion relation for the common-mode signals by considering inter-resonator coupling in the common-mode equivalent circuit model. Section IV discusses the effects of inter-resonator coupling and number of resonators on bandwidth enhancement and compares the common-mode filtering capabilities of CSRRs and DS-CSRRs. A methodology to designing CSRR or DS-CSRR common-mode filters and to estimating the achievable common-mode rejection bandwidth is presented in Section V. Section VI is focused on the characterization of two devices designed to demonstrate the selective mode suppression in DS-CSRR-based differential lines, and the enhanced rejection of the common mode in balanced filters loaded with DS-CSRRs. A comparison to other common mode filters is given in Section VII. Finally, the main conclusions are highlighted in Section VIII.

\section{Selective Common-Mode SupPression In DifFerential LiNES BY MEANS OF CSRRs: PRINCIPLE AND MODELING}

SRRs and CSRRs magnetically and electrically coupled, respectively, to transmission lines have been used for the implementation of stopband filters in microstrip and coplanar waveguide (CPW) technology [10]-[13]. In the vicinity of their resonance frequency, an array of electrically small SRRs/CSRRs excited with the magnetic/electric field in the axial direction (i.e., normal to the SRR or CSRR plane) behaves as an effective medium with negative permeability/permittivity [14]-[16]. Under these polarization conditions such media are opaque and signal propagation is inhibited around resonance. The stopband characteristics of transmission lines periodically loaded with SRRs or CSRRs have been interpreted as due to the negative effective permeability or permittivity of these one-dimensional (1-D) effective structures [2], [15]. However, the stopband behavior of these artificial lines can also be explained from circuit theory, since the SRRs and CSRRs (coupled to the lines) introduce transmission zeros.

Let us now discuss how CSRRs can be used to selectively suppress the even mode in microstrip differential transmission lines. As reported in [1], the strategy to suppress the common mode in microstrip differential lines, while keeping the fundamental (odd) mode unaffected, is to symmetrically etch an array
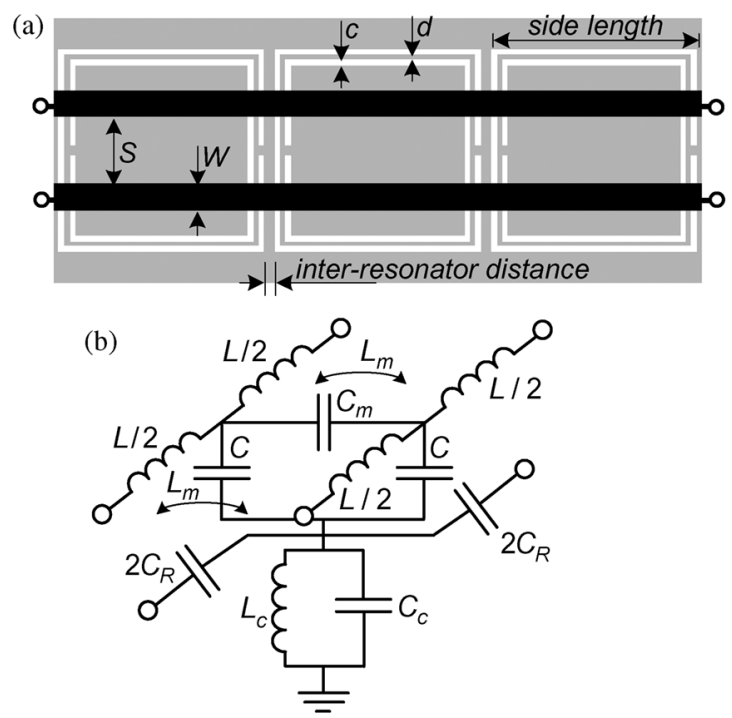

Fig. 1. (a) Typical topology of a microstrip differential transmission line with square-shaped CSRRs symmetrically etched in the ground plane to suppress the even mode. (b) Equivalent circuit model of the unit cell, including inter-resonator coupling. The ground plane is depicted in gray. The relevant dimensions are indicated.

of CSRRs in the ground plane, as shown in Fig. 1(a). The circuit model of the unit cell of this structure (including the electric coupling between adjacent resonators by means of a capacitor) is depicted in Fig. 1(b). As reported in [1], $L$ models the inductance of the lines, $C$ accounts for the electric coupling between the lines and the resonator, the CSRR is modeled by the parallel resonant tank, $L_{c}-C_{c}$, and $C_{m}$ and $L_{m}$ model the mutual capacitance and inductance between the coupled lines, respectively. Finally, $C_{R}$ models the electric coupling between adjacent resonators. In the model reported in [1], such inter-resonator coupling was neglected. However, our intention is to study the effects of such coupling on the rejection bandwidth for the common mode. Therefore, the introduction of $C_{R}$ in the model is necessary, and the result is a six-port network.

In view of Fig. 1(b), the differential signals are insensitive to the presence of the CSRRs, but these resonators prevent the transmission of the common mode at certain frequencies. The equivalent circuit models (unit cell) of the structure of Fig. 1(a) under even and odd mode excitation are depicted in Fig. 2(a) and (b), respectively. For the odd mode, the resonator is short-circuited to ground, and the resulting model is that of a conventional transmission line. For the even mode, we obtain the circuit of a conventional CSRR-loaded line [17], but with modified parameters and inter-resonator coupling (hence a four-port network). Thus, we do expect a stopband behavior for the common mode, enhanced by the presence of coupling between adjacent CSRRs (as will be shown later).

In terms of field distributions, it is well known that the CSRR (first resonance) can be excited by means of a time-varying axial electric field [2]. For the common mode, there is a strong density of electric field lines in the same direction below both lines. This causes CSRR excitation and hence a stopband. For the odd mode, the axial components of the electric field distribution exactly cancel if the structure is symmetric, (i.e., the gaps of the 


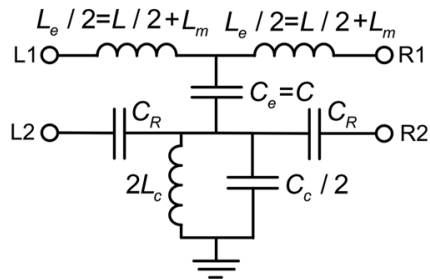

(a)

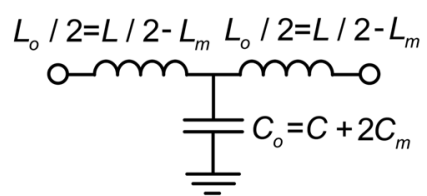

(b)
Fig. 2. Circuit model (unit cell) of the differential line loaded with CSRRs for (a) the even and (b) odd modes.

CSRR are aligned with the symmetry plane of the differential line), and the CSRR is not activated.

\section{COMMON-MODE DISPERSION RELATION}

Here, we infer the dispersion relation for the common mode of a differential line periodically loaded with CSRRs. Let us consider an infinite structure formed by cascading the four-port blocks that describe the unit cell of a microstrip differential line loaded with CSRRs for the common mode [Fig. 2(a)]. Let $V_{L 1}$, $V_{L 2}, I_{L 1}$, and $I_{L 2}$ be the voltages and currents at the ports ( 1 and 2 ) of the left-hand-side (subscript $L$ ) of the unit cell, and $V_{R 1}$, $V_{R 2}, I_{R 1}$, and $I_{R 2}$ the variables at the right-hand-side ports. From multiconductor line theory [18], [19], it follows that the variables at both sides of the unit block are related by

$$
\left(\begin{array}{c}
V_{L} \\
I_{L}
\end{array}\right)=\left(\begin{array}{ll}
A & B \\
C & D
\end{array}\right)\left(\begin{array}{c}
V_{R} \\
I_{R}
\end{array}\right)
$$

where $\boldsymbol{A}, \boldsymbol{B}, \boldsymbol{C}$ and $\boldsymbol{D}$ are $2 \times 2$ matrices while $\boldsymbol{V}_{\boldsymbol{L}}, \boldsymbol{I}_{\boldsymbol{L}}, \boldsymbol{V}_{\boldsymbol{R}}$ and $I_{R}$ are column vectors composed of the pair of port variables. As it follows from Bloch mode theory, the dispersion relation is obtained from the eigenmodes of the above system, which are inferred by forcing a given phase shift along the unit block, and setting the determinant of the resulting $4 \times 4$ matrix to be null:

$$
\operatorname{det}\left(\begin{array}{cc}
\boldsymbol{A}-e^{\gamma l} \cdot \boldsymbol{I} & \boldsymbol{B} \\
\boldsymbol{C} & \boldsymbol{D}-e^{\gamma l} \cdot \boldsymbol{I}
\end{array}\right)=0
$$

where $\boldsymbol{I}$ is the identity matrix, the phase-shift factor $e^{\gamma l}$ is the eigenvalue, $\gamma$ is the complex propagation constant, and $l$ is the unit cell length. For reciprocal, lossless, and symmetric networks, the eigenvalue solution can be simplified to the solution of the following equation [20], [21]:

$$
\operatorname{det}(\boldsymbol{A}-\cosh (\gamma l) \cdot \boldsymbol{I})=0 .
$$

The $\boldsymbol{A}$ matrix elements for the network of Fig. 2(a) can easily be calculated as

$$
\boldsymbol{A}=\left(\begin{array}{ll}
A_{11} & A_{12} \\
A_{21} & A_{22}
\end{array}\right)
$$

with

$$
\begin{aligned}
& A_{11}=1-L_{e} C_{e} \omega^{2} / 2 \\
& A_{12}=\frac{L_{e} C_{e} \omega^{2}}{2} \\
& A_{21}=-\frac{C_{e}}{C_{R}} \\
& A_{22}=1+\frac{C_{e}}{C_{R}}+\frac{C_{c}}{2 C_{R}}\left(1-\frac{1}{L_{c} C_{c} \omega^{2}}\right)
\end{aligned}
$$

where $\omega$ is the angular frequency. By introducing (4) into (3) and solving the determinant, we can obtain $\gamma l$. In this four-port network, there are two solutions (denoted later by $a$ and $b$ ) for $\gamma l$ :

$$
\cosh (\gamma l)=\frac{1}{2}\left(A_{11}+A_{22} \pm \sqrt{\left(A_{11}-A_{22}\right)^{2}+4 A_{12} A_{21}}\right) .
$$

The dispersion relation (6) will be used in Sections IV and V, respectively, to study the effects of inter-resonator coupling on bandwidth enhancement and to predict the maximum achievable bandwidth of periodic CSRR-loaded differential lines.

\section{BROADENING THE COMMON-MOdE REJECTION BANDWIDTH}

As pointed out in [1], there are three main strategies to enhance the rejection bandwidth in CSRR-based structures: 1) to tune the resonance frequency of several resonators in order to cover a wide band [10]; 2) to couple the resonators; and 3) to enhance the bandwidth of the individual resonators (and, obviously, a combination of the previous approaches). The multiple tuned resonators approach is efficient, but many particles are needed to cover a wide band if narrowband resonators are used. In the following subsections, the other two strategies for common-mode rejection enhancement in microstrip differential lines are discussed.

\section{A. Inter-Resonator Coupling}

The analysis of the dispersion relation is a convenient mean to gain insight on the rejection bandwidth for the common mode and, specifically, to analyze the effects of inter-resonator coupling on bandwidth enhancement. We have obtained the two modal propagation constants by considering the following element values: $L_{e}=6.3 \mathrm{nH}, C_{e}=1.1 \mathrm{pF}, L_{c}=2.1 \mathrm{nH}, C_{c}=$ $3.2 \mathrm{pF}$, and $C_{R}=0.1 \mathrm{pF}$ (i.e., those extracted from an electrically small CSRR-based structure considered in Section V). The results, shown in Fig. 3, indicate that, for each mode, there are regions where $\gamma l$ is purely real (evanescent mode), purely imaginary (propagating mode), or complex (complex mode). If complex modes are present, these modes must appear as conjugate pairs [8], and this is exactly the case in the region comprised between $1.42 \mathrm{GHz}$ and $1.61 \mathrm{GHz}$, that is $\alpha_{a}=\alpha_{b}$ and $\beta_{a}=-\beta_{b}$, where $\gamma=\alpha+\mathrm{j} \beta$. The forbidden band includes the region where complex modes are present (these modes do not carry net power [8], [22]-[24]), plus an additional region where $\beta_{a}=\beta_{b}=0, \alpha_{a} \neq 0$, and $\alpha_{b} \neq 0$ (between $1.61 \mathrm{GHz}$ 

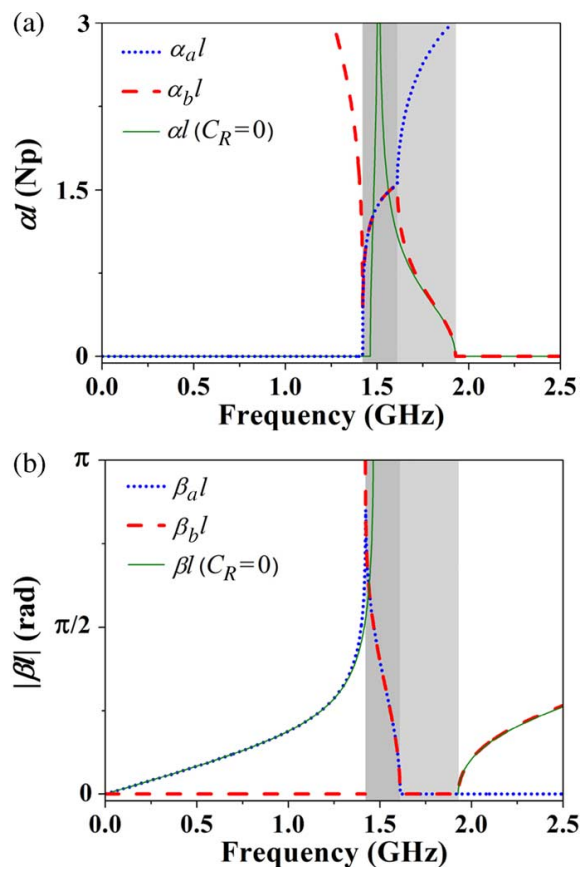

Fig. 3. (a) Attenuation constant and (b) magnitude of the phase constant of $\gamma l$ corresponding to the two modes of the network of Fig. 2(a). If inter-resonator coupling is cancelled $\left(C_{R}=0\right)$, the two modes degenerate in a single mode, also depicted. The evanescent and complex mode regions are highlighted in light and dark gray, respectively.

and $1.93 \mathrm{GHz}$ ) in which the modes are evanescent. As pointed out in [24], the pair of complex modes also inhibits signal propagation since such modes co-exist and have contra directional phase. The forward complex mode is related to microstrip wave propagation, whereas the backward complex mode resembles electroinductive waves [25] (the dispersion behavior is similar to that found in a shielded Sievenpiper structure [24]). The presence of coupling results in a wider stop band, as compared to the structure without electric coupling between adjacent resonators. This has been corroborated from the dispersion relation that results by forcing $C_{R}=0$ (also depicted in Fig. 3). However, by magnifying inter-resonator coupling (large value of $C_{R}$ ), the forbidden band does not substantially increase as compared with the case with $C_{R}=0.1 \mathrm{pF}$. Thus, inter-resonator coupling helps to broaden the stopband of the common mode in CSRR-loaded differential lines, but the sensitivity in bandwidth based on CSRR-to-CSRR coupling is limited, which makes apparent the convenience of implementing the common-mode rejection filters with broadband resonators.

The dispersion relation of common-mode filters described by a similar circuit to that of Fig. 2(a) is reported in [4], where the four-port $Z$-matrix of the circuit model and periodic boundary conditions related with the Bloch-Floquet theorem are used. Our reported approach is based on the transmission $A B C D$ matrix and multiconductor transmission line theory, and the complex modes are pointed out as the cause of rejection bandwidth enhancement for the common mode.

\section{B. Wideband Resonators: DS-CSRRs}

As was illustrated in Section IV-A, inter-resonator coupling enhances bandwidth but, in practice, such effect is limited.

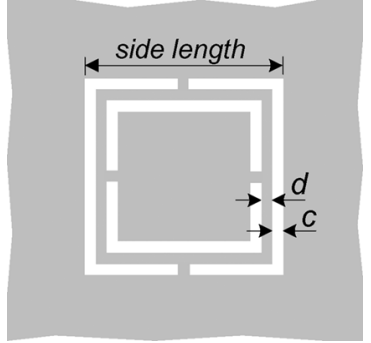

Fig. 4. Topology of a square-shaped DS-CSRR.

Therefore, it is important to increase the bandwidth of the individual resonators as much as possible. Here, we consider an alternative to that reported in [1] to obtaining wideband resonators.

In order to obtain a wide stopband response with a single CSRR coupled to a transmission line, it is necessary to increase the coupling capacitance between the line and the resonator and to reduce the CSRR inductance and capacitance. In [1], this was achieved by increasing the rings width and inter-rings space, with the result of a structure with tiny coupled slot rings. Under these circumstances, the CSRR is properly described by an $L C$ resonant tank capacitively coupled through the main line in a very narrow region in the vicinity of the transmission zero (nevertheless, it was demonstrated in [1] that, for CSRRs with narrow and closely spaced rings, the equivalent circuit of Fig. 2(a) is valid within a wide band).

As an alternative to obtain wideband resonators, the DS-CSRR can be considered [9] (see Fig. 4). Like the CSRR, the DS-CSRR is topologically symmetric, which makes it appropriate for the selective common-mode suppression. In comparison to a CSRR with the same dimensions and etched in the same substrate, the DS-CSRR has almost the same capacitance, but four times smaller inductance [9]. Hence, the DS-CSRR resonance frequency and electrical size are twice those of the CSRR. Since for a given (required) transmission zero, the DS-CSRR is larger than the CSRR, the coupling capacitance can also be larger, and, by virtue of a smaller inductance (as compared to that of a CSRR), we can obtain a wider bandwidth. Notice that since the DS-CSRR is electrically larger than the CSRR, it is expected that the circuit model of Fig. 2(a) provides an accurate description for the common mode over a wider band for the CSRR-loaded lines. This means that any prediction on common-mode rejection bandwidth based on the circuit model (Section V) is expected to be more accurate for CSRR-loaded lines. Nevertheless, it will be shown that approximate estimations can also be made by considering DS-CSRRs. Obviously, DS-CSRRs with wide and distant slot rings can also be considered to further enhancing the common-mode rejection bandwidth, but in this case the circuit model cannot be used for predicting the stopband (the same occurs with CSRRs with wide and distant slot rings). Indeed, the accuracy of the model depends not only on the electrical size of the particles, but also on the slot width and the inter-slot distance. The electrical size is intimately related to the coupling (and hence to the separation $d$ ) between the inner and outer slot rings. In practice, the minimum printable slot and strip width 
(in conventional PCB technology) is limited to 100 or $150 \mu \mathrm{m}$, and this dictates the frequency limits where the circuit model of Fig. 2(a) provides an accurate description of the structure.

To compare the effects of both particles on common-mode rejection bandwidth and to study the effects of increasing their number (filter order), we have designed several CSRR- and DS-CSRR-loaded differential lines. For a proper comparison, we have set $c$ and $d$ to the same value for both particles, specifically to our minimum nominal value that guarantees acceptable tolerance deviations in the actual values of the fabricated prototypes $(c=d=200 \mu \mathrm{m})$. In addition, the side length of the particles has been adjusted in order to obtain the required filter central frequency $f_{0}$. By means of the Agilent Momentum full-wave electromagnetic solver, we have computed the fractional rejection bandwidth for the common mode, $\mathrm{FBW}=\mathrm{BW} / f_{0}=\left|f_{1}-f_{2}\right| /\left(f_{1} \cdot f_{2}\right)^{1 / 2}$, where the upper and lower frequency limits of the stopband $f_{1}$ and $f_{2}$ have been considered to be those frequencies where the common-mode rejection level is $20 \mathrm{~dB}$ (losses have been excluded in these simulations). The results for different orders and central filter frequencies are shown in Fig. 5(a). As expected, the common-mode rejection bandwidth is wider for DS-CSRR-loaded differential lines. Despite the fact that, for a given central frequency an individual DS-CSRR is larger than a CSRR, if we compare CSRR- and DS-CSRR-loaded lines with the same area [see Fig. 5(b)], we see that the rejection bandwidth is wider for the latter. With these results, it can be concluded that DS-CSRRs exhibit a good balance between common-mode filter size and achievable bandwidth.

The results of Fig. 5 also show that the electrical size of the structures increases as the central frequency increases. That occurs because, in shifting the central frequency up, the particle dimensions have not been scaled down, that is, resonator side length has been decreased, but $c$ and $d$ have been kept constant (as well as the substrate height). It is also worth mentioning that the common-mode rejection bandwidth decreases as the central frequency increases. This aspect is attributed to a drastic decrease in the coupling capacitance between the differential line and the resonators due to the fact that the substrate thickness is not scaled down.

\section{COMmon-Mode FiLter Design}

\section{A. Filter Design}

The aim of this section is to provide a methodology for the design of microstrip differential lines with common-mode suppression and to predict the achievable rejection bandwidth on the basis of the common-mode equivalent circuit model and dispersion relation. For the reasons explained before, relative to the validity of the model of Fig. 2(a) and fabrication tolerances, we will consider $c=d=200 \mu \mathrm{m}$ (notice that this reduces the degrees of freedom and eases the common-mode filter design). We use square-shaped resonators (rather than circular) to enhance the electric coupling between the differential line and the resonators and between adjacent resonators as well. To further enhance inter-resonator coupling, the separation between adjacent resonators is also set to $200 \mu \mathrm{m}$. The differential line

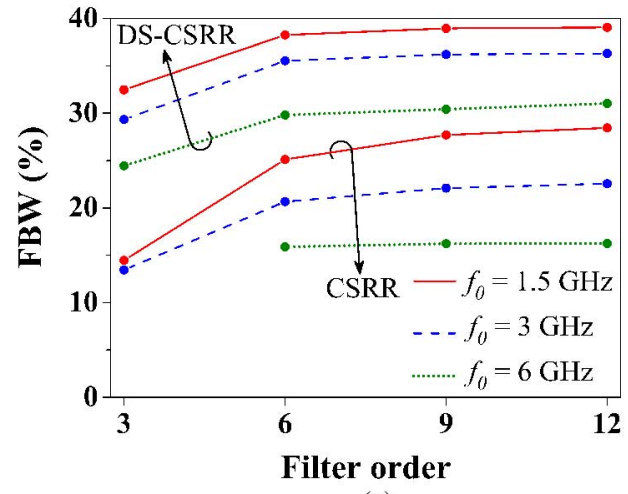

(a)

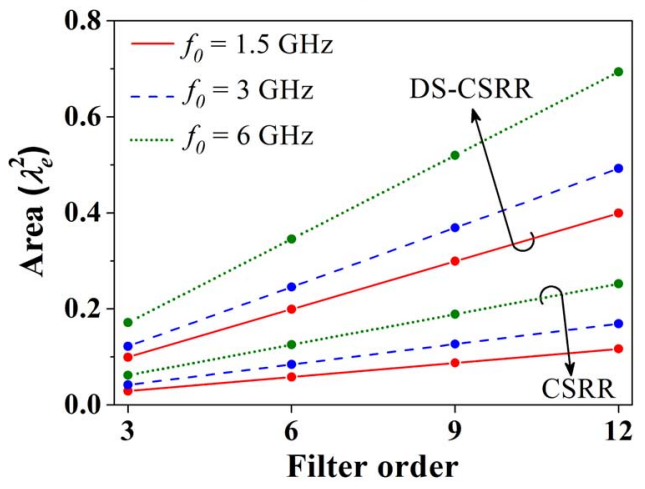

(b)

Fig. 5. (a) Fractional rejection bandwidth (FBW) at $-20 \mathrm{~dB}$ for the common mode given by electromagnetic simulation and (b) area as a function of the even mode guided wavelength $\gamma_{e}$ of CSRR- and DS-CSRR-loaded differential lines. Dimensions are: for the CSRRs and DS-CSRRs, $c=d=0.2 \mathrm{~mm}$, and inter-resonator distance $=0.2 \mathrm{~mm}$; for the CSRRs, side length $=7.3 \mathrm{~mm}$ $\left(f_{0}=1.5 \mathrm{GHz}\right), 4.3 \mathrm{~mm}\left(f_{0}=3 \mathrm{GHz}\right)$, and $2.6 \mathrm{~mm}\left(f_{0}=6 \mathrm{GHz}\right)$; for the DS-CSRRs, side length $=3.8 \mathrm{~mm}\left(f_{0}=1.5 \mathrm{GHz}\right), 7.5 \mathrm{~mm}\left(f_{0}=3 \mathrm{GHz}\right)$, and $4.3 \mathrm{~mm}\left(f_{0}=6 \mathrm{GHz}\right)$; for the differential line, $2 W+S=$ side length $2(2 c-d)+0.4 \mathrm{~mm}$ exhibiting a $50-\Omega$ characteristic impedance (odd mode). The considered substrate is the Rogers RO3010 with thickness $h=1.27 \mathrm{~mm}$, and dielectric constant $\varepsilon_{r}=10.2$.

is designed to exhibit an odd-mode characteristic impedance of $50 \Omega$. In order to achieve a strong electric coupling between the pair of lines and the resonator, the lines must be fitted inside the CSRR (or DS-CSRR) region and must be as wide as possible and, hence, as uncoupled as possible (line dimensions can easily be inferred from a transmission line calculator). The side length of the resonator is determined from the model of the CSRR reported in [17] (which gives $L_{c}$ and $C_{c}$ ) and the per-unit length capacitance of the coupled lines for the even mode (which gives $C_{e}$ ). The transmission zero frequency, given by

$$
f_{z}=\frac{1}{2 \pi \sqrt{2 L_{c}\left(C_{e}+C_{c} / 2\right)}}
$$

is adjusted to the required central filter frequency, and this provides the CSRR side length (for a DS-CSRR the side length can also be determined by taking into account that the inductance is four times smaller than the inductance of the CSRR). Obviously, optimization of the resonator side length in order to fit the required transmission zero is necessary (notice that the transmission zero frequency provides a reasonable estimate of the central filter frequency). 
TABLE I

EXTRACTED PARAMETERS AND MAXIMUM FRACTIONAL BANDWIDTH INFERRED FROM THE CIRCUIT MODEL

\begin{tabular}{c|ccccc|c}
\hline $\begin{array}{c}f_{0} \\
(\mathrm{GHz})\end{array}$ & $\begin{array}{c}L_{e} \\
(\mathrm{nH})\end{array}$ & $\begin{array}{c}C_{e} \\
(\mathrm{pF})\end{array}$ & $\begin{array}{c}L_{c} \\
(\mathrm{nH})\end{array}$ & $\begin{array}{c}C_{c} \\
(\mathrm{pF})\end{array}$ & $\begin{array}{c}C_{R} \\
(\mathrm{pF})\end{array}$ & $\begin{array}{c}F B W \\
(\%)\end{array}$ \\
\hline \multicolumn{7}{c}{ CSRR-loaded differential lines } \\
1.5 & 6.3 & 1.1 & 2.1 & 3.2 & 0.1 & 30.7 \\
3 & 4.2 & 0.5 & 1.0 & 2.0 & 0.06 & 26.4 \\
6 & 3.0 & 0.2 & 0.4 & 1.4 & 0.08 & 20.7 \\
\hline \multicolumn{7}{c}{ DS-CSRR-loaded differential lines } \\
1.5 & 15.9 & 1.6 & 1.0 & 8.6 & 0.64 & 32.6 \\
3 & 9.0 & 0.8 & 0.45 & 4.8 & 0.18 & 29.4 \\
6 & 6.9 & 0.3 & 0.15 & 3.6 & 0.1 & 23.6 \\
\hline
\end{tabular}

With the previous procedure, the common-mode filter dimensions are perfectly determined. To predict the maximum achievable bandwidth, i.e., the bandwidth obtained by considering an infinite number of cells, the dispersion relation is used. However, since optimization at the layout level is required, it is necessary to extract the parameters of the circuit model following the procedure reported in [26], where the element values are obtained from certain mapping conditions such as the transmission zero frequency and the intercept of the reflection coefficient $\left(S_{11}\right)$ with the unit resistance circle. Thus, $L_{e}, C_{e}, L_{c}$, and $C_{c}$ are first extracted by considering a single cell structure, and then $C_{R}$ is adjusted to fit the electromagnetic simulation of an order- 2 common-mode filter. Once the circuit parameters are known, (6) can be evaluated, and the common-mode stopband can be determined.

Following the previous approach, we have obtained the maximum achievable rejection bandwidth for different CSRR and DS-CSRR common-mode filters. To compare with full-wave electromagnetic simulations, we have considered those central frequencies and that substrate of Fig. 5. The extracted circuit parameters and estimated fractional bandwidths are shown in Table I. By comparing the maximum fractional bandwidths predicted by the reported approach with the saturation values of Fig. 5(a), we can conclude that the reported approach is more accurate for CSRR-loaded lines, as expected. Fig. 6 compares the circuit and electromagnetic simulation (common mode) of the order-1, -2 , and -3 CSRR-based common-mode filters designed to exhibit a central frequency (actually the transmission zero for the single-stage structure) of $1.5 \mathrm{GHz}$. Note that, in the circuit simulation, the inter-resonator capacitance at input and output ports have been left opened since the CSRRs of the input and output cells are not externally fed, resulting in a two-port circuit. There is good agreement between the circuit and electromagnetic simulation.

As can be seen in Fig. 5(a), six resonators are sufficient to nearly achieve the maximum rejection bandwidth. Obviously, filter size can be reduced by decreasing the number of resonators but at the expense of a reduced common mode rejection bandwidth. Thus, following a systematic approach based on the circuit model of the common mode, we can infer whether a specified rejection bandwidth and central frequency can be roughly fulfilled or not. If the required bandwidth is wider, we are forced to consider resonators with wider slots $(c)$ and inter-slot distance $(d)$, or, alternatively, multiple tuned resonators. In this case, however, filter design and maximum bandwidth estimation

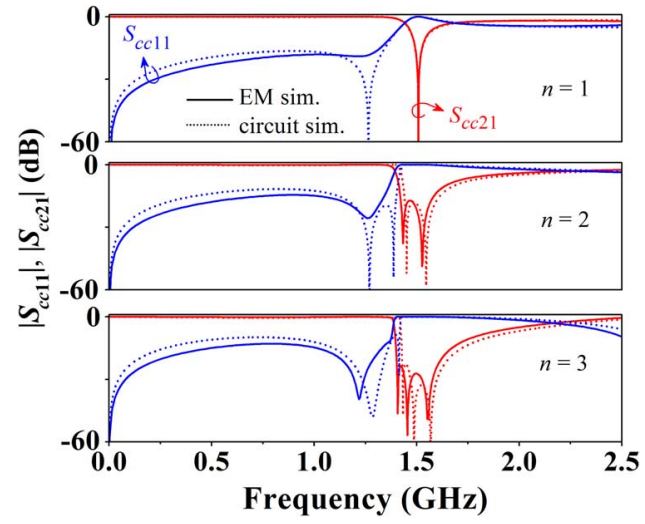

Fig. 6. Common-mode return loss $\left|S_{c c 11}\right|$ and insertion loss $\left|S_{c c 21}\right|$ given by the electromagnetic and circuit simulation for the order-1, -2 , and -3 commonmode filters (1.5 GHz central frequency) based on CSRRs. Dimensions and substrate are indicated in the caption of Fig. 5. Circuit parameters are given in Table I.

are not so straightforward. Notice that the reported approach allows us to infer the maximum achievable rejection bandwidth, rather than setting the common mode rejection bandwidth to a given value.

\section{B. Discussion on the Rejection Bandwidth Definition}

Finally, it is important to discuss if the meaning of the maximum fractional bandwidth inferred from the dispersion relation actually corresponds to the $-20 \mathrm{~dB}$ bandwidth of a structure with a large number of cells. Indeed, the dispersion relation provides the frequency regions where signal propagation is allowed or forbidden for an infinite number of cells. For a finite number of cells, the most relevant effects that may occur are impedance mismatch in the allowed bands or small rejection within the forbidden bands. Therefore, an analysis to determine the rejection level that properly defines the forbidden band is very convenient.

It is well known that the transmission coefficient in two-port periodic structures exhibits ripple in the allowed bands. Such ripple is caused by impedance mismatch, and maximum transmission is produced at those frequencies where either the Bloch impedance is matched to the impedance of the ports (impedance matching) or the electrical length is a multiple of $\pi$ (phase matching). Between adjacent transmission peaks, the transmission coefficient is a minimum at those frequencies where the phase is an odd multiple of $\pi / 2$. The transmission coefficient at these frequencies of minimum transmission can easily be obtained according to [27]

$$
f_{\text {env }}(\omega)=10 \log \left\{1-\left|\frac{Z_{B}^{2}(\omega)-Z_{o}^{2}}{Z_{B}^{2}(\omega)+Z_{o}^{2}}\right|^{2}\right\} .
$$

Indeed, the previous function gives the envelope of the transmission coefficient in the allowed bands. Expression (8) corresponds to a two-port structure, where $Z_{B}(\omega)$ is the Bloch impedance and $Z_{O}$ the reference impedance of the ports $\left(Z_{o}=50 \Omega\right)$. However, the structure under analysis is described by a circuit model with two input and two output ports [Fig. 2(a)]. In a two-port structure, the Bloch impedance is obtained by the ratio between the eigenvector variables (voltage and current). For the circuit of Fig. 2(a), the pair of eigenvectors 
( $\left[V_{1 a}, V_{2 a}, I_{1 a}, I_{2 a}\right]$ and $\left.\left[V_{1 b}, V_{2 b}, I_{1 b}, I_{2 b}\right]\right)$ can be obtained by using (1) and (6), and we can infer from them the characteristic impedance matrix as follows [18]:

$$
Z_{c}=V_{a b} \cdot I_{a b}^{-1}
$$

where $V_{\boldsymbol{a b}}$ and $I_{\boldsymbol{a} \boldsymbol{b}}$ are $2 \times 2$ matrices formed by column vectors composed of the eigenvector voltages and currents

$$
\begin{aligned}
\boldsymbol{V}_{a b} & =\left(\begin{array}{ll}
V_{1 a} & V_{1 b} \\
V_{2 a} & V_{2 b}
\end{array}\right) \\
\boldsymbol{I}_{\boldsymbol{a b}} & =\left(\begin{array}{ll}
I_{1 a} & I_{1 b} \\
I_{2 a} & I_{2 b}
\end{array}\right) .
\end{aligned}
$$

The diagonal elements of $\boldsymbol{Z}_{\boldsymbol{c}}\left(Z_{11}\right.$ and $\left.Z_{22}\right)$ are simply the ratios between the voltage and current in the corresponding port when the other port is left open, whereas the anti-diagonal elements determine the effects of the current injected in one port on the voltage in the other port when it is open. Notice that $Z_{11}$ and $Z_{22}$ are not the ratios of the voltage and current at ports 1 and 2 for each eigenvector. Let us denote such voltage-to-current ratios for port 1 as $Z_{B 1 a}$ and $Z_{B 1 b}$, where $a$ and $b$ discriminate each mode. If we now compare $Z_{11}$ with $Z_{B 1 a}$ and $Z_{B 1 b}$ (Fig. 7), it can be appreciated that $Z_{11} \approx Z_{B 1 a}$ to the left of the forbidden band, whereas $Z_{11} \approx Z_{B 1 b}$ to the right of that band. This means that, in the allowed bands, the propagation in an infinite structure resulting by cascading the networks of Fig. 2(a) with port L2 of the first cell left opened, can be described as if it was a two-port network that supports the eigenvector composed of the variables referred to port 1 (mode $a$ and $b$ to the left and right of the stopband, respectively) and Bloch impedance given by $Z_{11}$. This approximation is valid as long as mode mixing is negligible, as occurs in the whole allowed frequency region except in a narrow band to the left of the stopband, where modes $a$ and $b$ coexist (this leads to a complex value of $Z_{11}$ in that region).

From previous statements, it follows that the envelope function of the transmission coefficient of any finite structure with ports L2 and R2 of the first and last cell, respectively, left open (actual conditions), can be approximated by means of (8), considering $Z_{B}=Z_{11}$. The envelope function in the allowed bands inferred through (8) with $Z_{11}$ of Fig. 7 is compared with the response of a 12-cell structure in Fig. 8. It is confirmed that we can use (8) to obtain the envelope function to a very good approximation.

With regard to the maximum bandwidth, it coincides with the region delimited by those frequencies where the envelope function exhibits a vertical slope. This vertical slope is clearly achieved at a rejection level of $20 \mathrm{~dB}$. Hence, this is a good rejection level to define the common-mode rejection bandwidth. We would like to emphasize that the proposed procedure to infer the envelope function is approximate. However, (8) provides very good results because the coupling capacitance between resonators, $C_{R}$, is necessarily small in the considered structures. If this condition is not fulfilled, mode mixing is more pronounced, and the envelope function that results when the ports L2 and R2 of the input and output cell, respectively, are opened, cannot be inferred following this simple procedure.
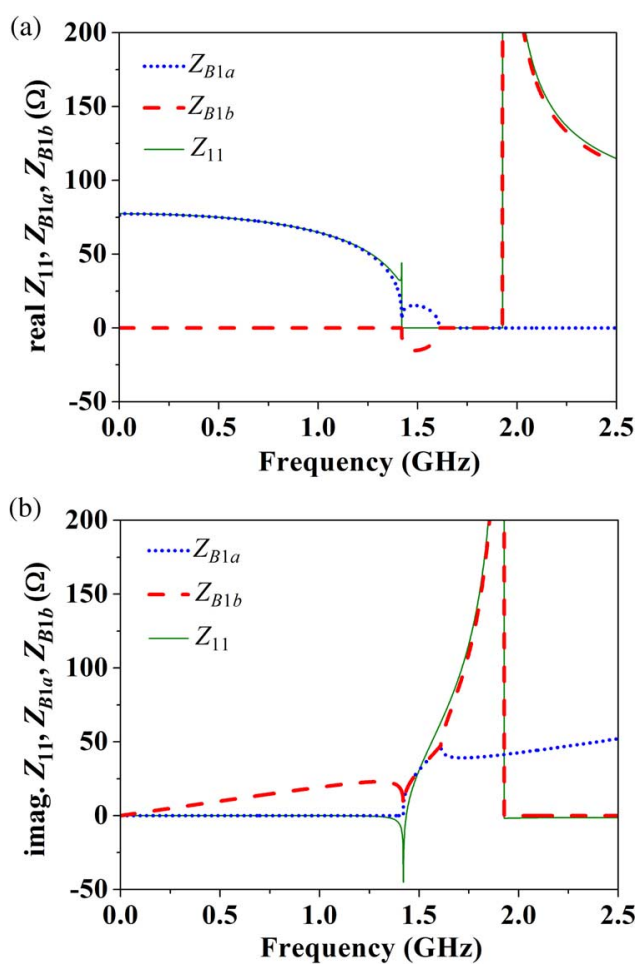

Fig. 7. (a) Real and (b) imaginary parts of $Z_{11}, Z_{B 1 a}$, and $Z_{B 1 b}$. The considered circuit parameters [refer to Fig. 2(a)] are those corresponding to the CSRR common-mode filter with $1.5-\mathrm{GHz}$ central frequency (i.e., those indicated in the first row of Table I).

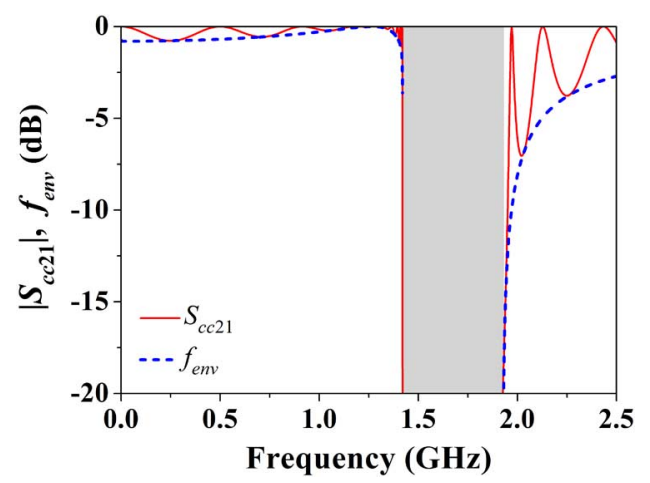

Fig. 8. Frequency response of the 12-cell structure resulting by cascading the four-port networks of Fig. 2(a) with L2 and R2 ports of stages 1 and 12, respectively, left opened, and envelope function in the allowed bands. The forbidden band is depicted in gray.

\section{EXPERIMENTAL VALIDATION AND APPLICATIONS}

Two application examples of the considered structures are presented in this section: 1) a DS-CSRR-loaded differential line with common-mode suppression and 2) a differential (balanced) bandpass filter with common-mode rejection enhanced by the presence of DS-CSRRs.

\section{A. Differential Line With Common-Mode Rejection}

Here, the common-mode suppression in microstrip differential lines loaded with DS-CSRRs is experimentally validated. We have designed a common-mode filter similar to that reported in [1], but in this case by using DS-CSRRs with tightly coupled slot rings. The target is to implement a common-mode 

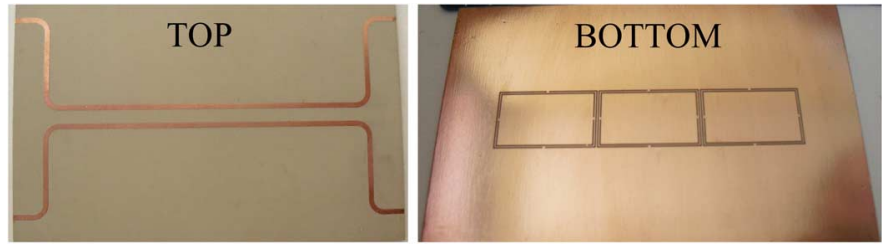

Fig. 9. Photograph of the designed differential line with common-mode suppression based on DS-CSRRs. Dimensions are: for the DS-CSRRs, $c=d=$ $0.2 \mathrm{~mm}$, longitudinal side length $=17.6 \mathrm{~mm}$, and transverse side length $=$ $10.8 \mathrm{~mm}$; inter-resonator distance $=0.2 \mathrm{~mm}$; for the differential line, $W=1 \mathrm{~mm}$, and $S=2.5 \mathrm{~mm}$. The considered substrate is the Rogers $R O 3010$ with thickness $h=1.27 \mathrm{~mm}$, dielectric constant $\varepsilon_{r}=10.2$, and loss tangent $\tan \delta=0.0023$

filter roughly centered at $1.35 \mathrm{GHz}$ and exhibiting at least $35 \%$ fractional bandwidth (at 20-dB rejection level). According to the previous methodology, these specifications cannot be fulfilled by using CSRRs with $c=d=200 \mu \mathrm{m}$. However, it is possible to achieve these filter requirements by means of DS-CSRRs. Indeed, the estimated maximum bandwidth for a common-mode filter centered at $1.35 \mathrm{GHz}$ has been found to be $37.3 \%$, but we do expect a larger value since the model tends to slightly underestimate the maximum achievable bandwidth for DS-CSRR-loaded lines [this can be appreciated by comparing Table I and Fig. 5(a)]. Moreover, for comparison purposes, a rectangular-shaped DS-CSRR has been considered with its transverse side length identical to that of the CSRR reported in [1]. This favors the electric coupling between the pair of lines and the DS-CSRRs and, hence, the common-mode stopband expansion (the reason is that the DS-CSRR longitudinal side is longer than the transverse one, and this increases the coupling capacitance $C_{e}$ as compared with that of a square-shaped DS-CSRR with identical transmission zero frequency). The longitudinal side length is thus the single design parameter, and this has been determined following the same approach applied to square-shaped particles (the geometrical parameters of the structure are given in the caption of Fig. 9).

The photograph and frequency response of the device, an order-3 common-mode filter, are depicted in Figs. 9 and 10, respectively (this filter order has been found to be sufficient to satisfy the bandwidth requirements). As can be seen, the differential signal is almost unaltered whilst the common mode is rejected within a fractional bandwidth $(41 \%)$ comparable to that achieved in [1] by using CSRRs with wide and widely spaced rings. The DS-CSRR-based structure is a bit larger than that reported in [1], but the design has been done following the systematic procedure explained in the previous section.

Fig. 11 shows the measured differential eye diagrams with the excitation of $0.2-\mathrm{V}$ amplitude in $2.5 \mathrm{~Gb} / \mathrm{s}$ for the differential line of Fig. 9 with and without DS-CSRRs. The eye diagram quality in terms of eye height, eye width, jitter, and eye opening factor is compared for these two structures (see Table II). According to these results, the presence of the DS-CSRRs does not significantly degrade the differential mode. The peak-to-peak jitter varies notably, but it is still within very acceptable limits for the DS-CSRR-based structure. Moreover, the eye opening factor, which measures the ratio of eye height and eye amplitude, is identical.
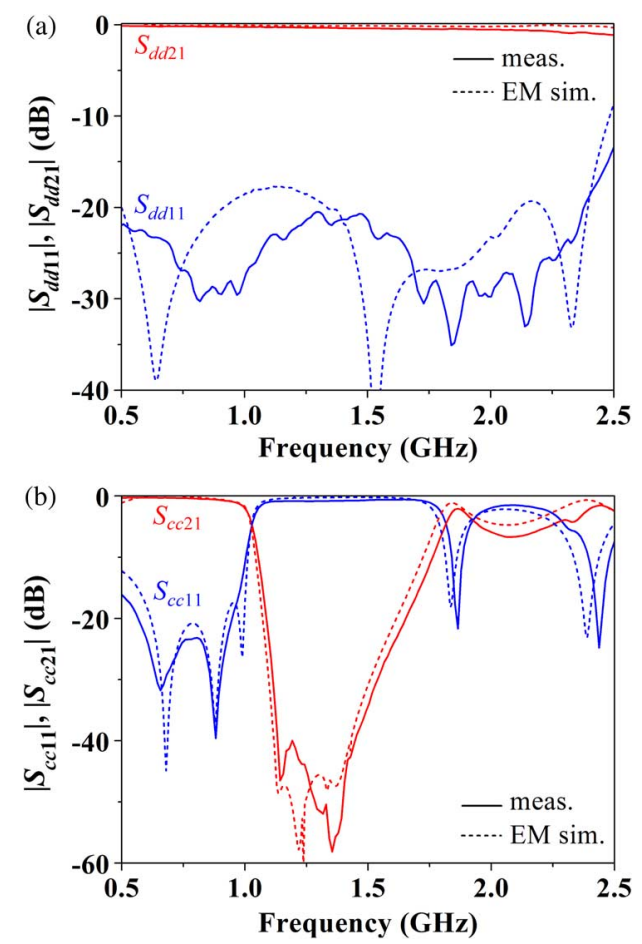

Fig. 10. (a) Differential-mode return loss $\left|S_{d d 11}\right|$ and insertion loss $\left|S_{d d 21}\right|$ and (b) common-mode return loss $\left|S_{c c 11}\right|$ and insertion loss $\left|S_{c c 21}\right|$ for the structure of Fig. 9.
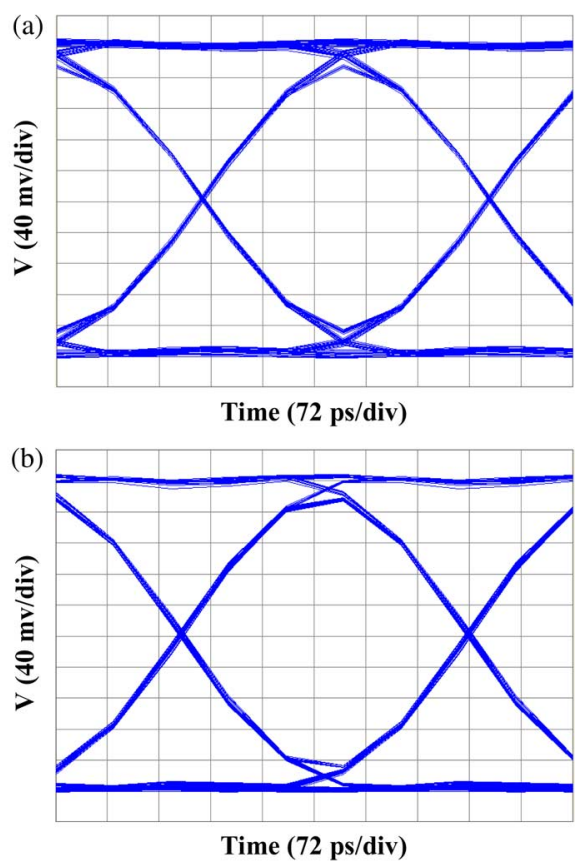

Fig. 11. Measured differential eye diagrams for the differential line of Fig. 9 (a) with and (b) without DS-CSRRs. The eye diagram in (a) reveals that the common-mode rejection does not degrade the differential signals.

\section{B. Differential Bandpass Filter With Common-Mode Rejection}

Differential bandpass filters with common-mode rejection have been reported in the literature [28]-[32]. Here, we report a balanced filter consisting of a pair of coupled stepped-impedance resonators (SIRs) fed by a differential line, 
TABLE II

MEASURED EYE PARAMETERS

\begin{tabular}{l|cc}
\hline & With DS-CSRRs & Without DS-CSRRS \\
\hline Eye height & $278 \mathrm{mV}$ & $281 \mathrm{mV}$ \\
Eye width & $371 \mathrm{ps}$ & $383 \mathrm{ps}$ \\
Jitter (PP) & $29.3 \mathrm{ps}$ & $16.9 \mathrm{ps}$ \\
Eye opening factor & 0.76 & 0.76 \\
\hline
\end{tabular}

(a)

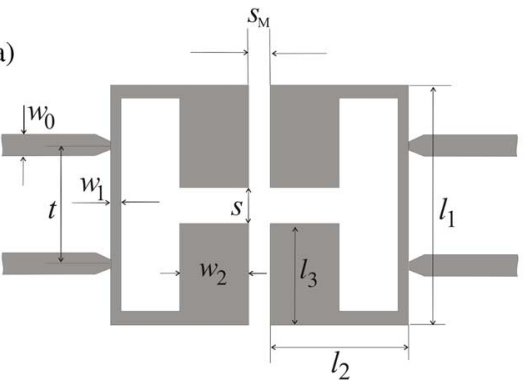

(b)
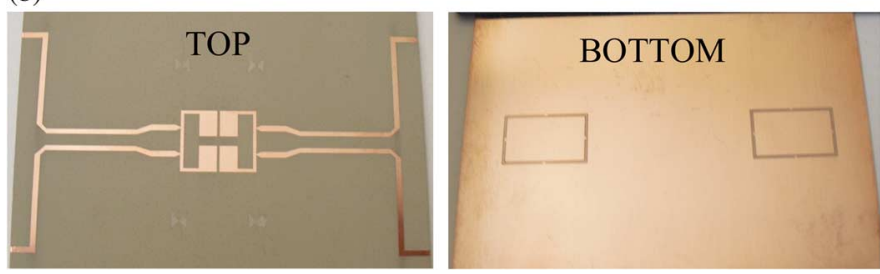

Fig. 12. (a) Layout and (b) photograph of the designed differential bandpass filter with improved common-mode rejection. Dimensions are: for the DS-CSRRs, $c=d=0.2 \mathrm{~mm}$, longitudinal side length $=17.6 \mathrm{~mm}$, and transverse side length $=10.8 \mathrm{~mm}$; for the differential line, $W=1 \mathrm{~mm}$, and $S=2.5 \mathrm{~mm}$; for the differential filter, $S_{M}=0.5 \mathrm{~mm}, s=2 \mathrm{~mm}$, $w_{0}=1.2 \mathrm{~mm}, w_{1}=0.7 \mathrm{~mm}, w_{2}=3.7 \mathrm{~mm}, l_{1}=12.8 \mathrm{~mm}, l_{2}=7.5 \mathrm{~mm}$, $l_{3}=5.4 \mathrm{~mm}$, and $t=5.5 \mathrm{~mm}$. The considered substrate is the Rogers RO3010 with thickness $h=1.27 \mathrm{~mm}$, dielectric constant $\varepsilon_{r}=10.2$, and loss tangent $\tan \delta=0.0023$.

as shown in the layout of Fig. 12(a) (other balanced filters based on SIRs have been reported in [33] and [34]). The filter by itself rejects the common mode due to the symmetry of the structure, since the symmetry plane of the resonator exhibits an electric wall at the first SIR resonance. Therefore, such resonators cannot be excited by means of common-mode signals, and the even mode is reflected back to the source due to the presence of the slots between the pair of SIRs. However, the rejection level of the common mode in the region of interest is very limited since it depends on the distance between resonators, and such inter-resonator distance is dictated by filter specifications. Next, we show that the common mode can be further rejected by introducing (cascading) DS-CSRRs.

The proposed differential filter is a second-order Chebyshev bandpass filter with a central frequency of $1.37 \mathrm{GHz}$, a fractional bandwidth of $10 \%$, and $0.1-\mathrm{dB}$ ripple. The considered substrate is the Rogers RO3010 with thickness $h=1.27 \mathrm{~mm}$, and dielectric constant $\varepsilon_{r}=10.2$. With these specifications and substrate, the layout of the filter is that depicted in Fig. 12(a) (the design of the filter has been done following the procedure described in [35]).

The frequency response of the filter (differential mode, $S_{d d 21}$ and $\left.S_{d d 11}\right)$ is shown in Fig. 13. The common-mode insertion loss $\left(S_{c c 21}\right)$, also depicted in the figure, exhibits a rejection

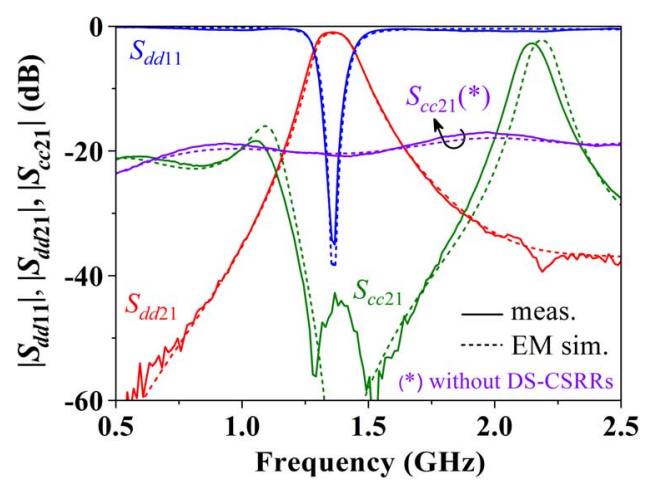

Fig. 13. Simulated and measured differential-mode return loss $\left|S_{d d 11}\right|$ and insertion loss $\left|S_{d d 21}\right|$, and common-mode insertion loss $\left|S_{c c 21}\right|$ for the structure of Fig. 12(b) with and without the presence of the DS-CSRRs.

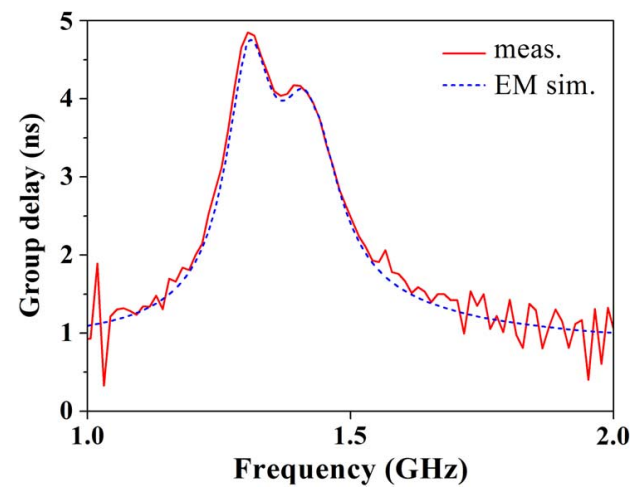

Fig. 14. Group delay for the differential filter of Fig. 12(b).

level of about $20 \mathrm{~dB}$ in the passband region. In order to enhance the common-mode rejection, we have cascaded two identical DS-CSRRs as shown in Fig. 12(b). Such DS-CSRRs have been designed to generate a stopband for the common mode in the passband region of the differential filter and are identical to those of Fig. 9. The presence of the DS-CSRRs does not affect the filter response (odd mode). However, by merely introducing two DS-CSRRs, the common mode rejection is roughly increased up to $50 \mathrm{~dB}$ in the region of interest (see Fig. 13). To completely characterize the differential filter, the group delay for the differential mode is shown in Fig. 14. These results point out that DS-CSRRs provide an efficient path to enhance the common mode noise rejection in balanced filters.

\section{COMPARISON With OTHER COMMON-MOdE FILTERS}

The fabricated differential line with common mode suppression of Fig. 9 is compared with that presented in [1] and with other reported differential lines in Table III. The physical dimensions given in the table correspond to the active area (defected ground area in the defected ground structures), and the guided wavelength $\gamma_{e}$ is the one corresponding to the even mode. As compared with other approaches, our CSRR and DS-CSRR based differential lines exhibit a competitive combination of size, bandwidth and rejection level. The filter reported in [4] is very competitive in terms of size and bandwidth, but the maximum rejection level is moderate. Moreover, such a common-mode filter is based on a multilayer structure, and this has extra cost and complexity. The filters reported in [5] and [6] 
TABLE III

COMParison of SEVERAL DifFERENTIAL Lines With COMMON-ModE SUPPRESSION

\begin{tabular}{c|ccc|ccc}
\hline Ref. & $\begin{array}{c}\text { Length } \\
\left(\lambda_{e}\right)\end{array}$ & $\begin{array}{c}\text { Width } \\
\left(\lambda_{e}\right)\end{array}$ & $\begin{array}{c}\text { Area } \\
\left(\lambda_{e}^{2}\right)\end{array}$ & $-10 \mathrm{~dB}$ & $\begin{array}{c}\text { FBW }(\%) \\
-20 \mathrm{~dB}\end{array}$ & $-40 \mathrm{~dB}$ \\
\hline$[4]$ & 0.26 & 0.16 & 0.04 & 60 & 32 & - \\
{$[5]$} & 0.76 & 0.47 & 0.36 & 70 & 53 & - \\
{$[6]$} & 0.44 & 0.44 & 0.19 & 87 & - & - \\
{$[1]$} & 0.43 & 0.14 & 0.06 & 54 & 37 & 14 \\
This work & 0.64 & 0.13 & 0.08 & 51 & 41 & 23 \\
\hline
\end{tabular}

have larger dimensions than our CSRR- and DS-CSRR-based filters do. The filter reported in [6] exhibits a huge stopband considering a $-10-\mathrm{dB}$ bandwidth, but, again, the maximum rejection level is poor as compared with that of our proposed filters.

In summary, the proposed common-mode suppression strategy is technologically simple and low cost (only two metal levels are used), and the resulting common-mode rejection filters are compact and provide wide and high-rejection stopbands, with a simple and systematic design procedure.

\section{CONCLUSION}

It has been demonstrated that CSRRs and DS-CSRRs are useful to selectively suppress the even (common) mode in microstrip differential lines. The lumped element equivalent circuit model (unit cell) of a differential line loaded with an array of symmetrically etched CSRRs (or DS-CSRRs) has been reported. From symmetry properties and the magnetic and electric wall concepts, the circuit models under even- and odd-mode excitation have also been obtained. Such models have been used to support the conclusions relative to the selective mode suppression (i.e., the suppression of the even mode maintaining the integrity of the differential signals) inferred from symmetry analysis and field distributions.

Since the common-mode rejection bandwidth is a key parameter to common mode filters design, the main strategies for bandwidth enhancement have been considered. Concerning inter-resonator coupling, it has been justified from Bloch mode theory that bandwidth enhancement in common mode filters with tightly coupled resonators is related to the presence of complex waves. Although inter-resonator coupling can enhance the bandwidth, this effect is limited by technological restrictions and, therefore, the use of intrinsically wideband resonators is essential to achieve competitive common mode rejection bands. For this reason, DS-CSRRs have been considered in this work.

A simple methodology for common-mode filter design (valid for CSRR and DS-CSRR) has been proposed, and several periodic common-mode filters with different central frequency and order have been designed and compared. Clearly, DS-CSRRs are more efficient in terms of rejection bandwidth, though the prediction of the maximum achievable bandwidth is more accurate for CSRR-loaded lines. It has also been found that the common-mode rejection bandwidth roughly saturates for order- 6 filters.

The theory has been validated against measurements through the fabrication of a DS-CSRR-loaded differential transmission line and a balanced bandpass filter with common-mode suppression. In the DS-CSRR-loaded line, a measured common-mode rejection level better than $20 \mathrm{~dB}$ within a $41 \%$ fractional bandwidth has been obtained (whilst preserving the integrity of the differential signal). The reported differential bandpass filter exhibits a measured common-mode rejection better than $40 \mathrm{~dB}$ in a 34\% bandwidth (including the differential passband region) thanks to the presence of the DS-CSRRs, which do not affect the differential filter performance.

\section{REFERENCES}

[1] J. Naqui, A. Fernández-Prieto, M. Durán-Sindreu, J. Selga, F. Medina, F. Mesa, and F. Martín, "Split rings-based differential transmission lines with common-mode suppression," in IEEE MTT-S Int. Microw. Symp. Dig., Baltimore, MD, Jun. 2011.

[2] F. Falcone, T. Lopetegi, J. D. Baena, R. Marqués, F. Martín, and M. Sorolla, "Effective negative- $\varepsilon$ stop-band microstrip lines based on complementary split ring resonators," IEEE Microw. Wireless Compon. Lett., vol. 14, no. 6, pp. 280-282, Jun. 2004.

[3] B. C. Tseng and L. K. Wu, "Design of miniaturized common-mode filter by multilayer low-temperature co-fired ceramic," IEEE Trans. Electromagn. Compat., vol. 46, no. 4, pp. 571-579, Nov. 2004.

[4] C.-H. Tsai and T.-L. Wu, "A broadband and miniaturized commonmode filter for gigahertz differential signals based on negative-permittivity metamaterials," IEEE Trans. Microw. Theory Tech., vol. 58, no. 1, pp. 195-202, Jan. 2010.

[5] W. T. Liu, C.-H. Tsai, T.-W. Han, and T.-L. Wu, "An embedded common-mode suppression filter for $\mathrm{GHz}$ differential signals using periodic defected ground plane," IEEE Microw. Wireless Compon. Lett., vol. 18, no. 4, pp. 248-250, Apr. 2008.

[6] S.-J. Wu, C.-H. Tsai, T.-L. Wu, and T. Itoh, "A novel wideband common-mode suppression filter for gigahertz differential signals using coupled patterned ground structure," IEEE Trans. Microw. Theory Techn., vol. 57, no. 4, pp. 848-855, Apr. 2009.

[7] G. V. Eleftheriades, "Analysis of bandwidth and loss in negative-refractive-index transmission-line (NRI-TL) media using coupled resonators," IEEE Microw. Wireless Compon. Lett., vol. 17, no. 6, pp. 412-414, Jun. 2007.

[8] F. A. Fernández, Y. Lu, J. B. Davies, and S. Zhu, "Finite element analysis of complex modes in inhomogeneous waveguides," IEEE Trans. Magn., vol. 29, pp. 1601-1604, Mar. 1993.

[9] R. Marqués, J. D. Baena, J. Martel, F. Medina, F. Falcone, M. Sorolla, and F. Martín, "Novel small resonant electromagnetic particles for metamaterial and filter design," in Proc. Int. Conf. Electromagn. Adv. Applications, Torino, Italy, Sep. 8-12, 2003, pp. 439-443.

[10] F. Martín, F. Falcone, J. Bonache, T. Lopetegi, R. Marqués, and M. Sorolla, "Miniaturized CPW stop band filters based on multiple tuned split ring resonators," IEEE Microw. Wireless Compon. Lett., vol. 13, no. 12, pp. 511-513, Dec. 2003.

[11] J. García-García, J. Bonache, F. Falcone, J. D. Baena, F. Martín, I. Gil, T. Lopetegi, M. A. G. Laso, A. Marcotegui, R. Marqués, and M. Sorolla, "Stepped-impedance low pass filters with spurious passband suppression,” Electron. Lett., vol. 40, pp. 881-883, Jul. 2004.

[12] J. García-García, F. Martín, F. Falcone, J. Bonache, I. Gil, T. Lopetegi, M. A. G. Laso, M. Sorolla, and R. Marqués, "Spurious passband suppression in microstrip coupled line bandpass filters by means of split ring resonators," IEEE Microw. Wireless Compon. Lett., vol. 14, no. 9, pp. $416-418$, Sep. 2004.

[13] J. García-García, F. Martín, F. Falcone, J. Bonache, J. D. Baena, I. Gil, E. Amat, T. Lopetegi, M. A. G. Laso, J. A. Marcotegui, M. Sorolla, and R. Marqués, "Microwave filters with improved stop band based on sub-wavelength resonators," IEEE Trans. Microw. Theory Tech., vol. 53, no. 6, pp. 1997-2006, Jun. 2005.

[14] D. R. Smith, W. J. Padilla, D. C. Vier, S. C. Nemat-Nasser, and S. Schultz, "Composite medium with simultaneously negative permeability and permittivity," Phys. Rev. Lett., vol. 84, pp. 4184-4187, May 2000 . 
[15] F. Martín, F. Falcone, J. Bonache, R. Marqués, and M. Sorolla, "Split ring resonator based left handed coplanar waveguide," Appl. Phys. Lett., vol. 83, pp. 4652-4654, Dec. 2003.

[16] F. Falcone, T. Lopetegi, M. A. G. Laso, J. D. Baena, J. Bonache, R. Marqués, F. Martín, and M. Sorolla, "Babinet principle applied to the design of metasurfaces and metamaterials," Phys. Rev. Lett., vol. 93, p. 197401, Nov. 2004.

[17] J. D. Baena, J. Bonache, F. Martín, R. Marqués, F. Falcone, T. Lopetegi, M. A. G. Laso, J. García, I. Gil, and M. Sorolla, "Equivalent circuit models for split ring resonators and complementary split rings resonators coupled to planar transmission lines," IEEE Trans. Microw. Theory Tech., vol. 53, no. 4, pp. 1451-1461, Apr. 2005.

[18] R. Mongia, I. Bahl, and P. Barthia, "Multiconductor transmission lines," in RF and Microwave Coupled Line Circuits. Norwood, MA: Artech House, 1999

[19] S. Gupta, A. Parsa, E. Perret, R. V. Snyder, R. J. Wenzel, and C. Caloz, "Group-delay engineered noncommensurate transmission line all-pass network for analog signal processing," IEEE Trans. Microw. Theory Tech., vol. 58, no. 9, pp. 2392-2407, Sep. 2010.

[20] J. Shekel, "Matrix analysis of multi-terminal transducers," Proc. IRE, vol. 42, no. 5, pp. 840-847, May 1954.

[21] R. Islam, M. Zedler, and G. V. Eleftheriades, "Modal analysis and wave propagation in finite 2-D transmission-line metamaterials," IEEE Trans. Antennas Propag., vol. 59, no. 5, pp. 1562-1570, May 2011

[22] U. Crombach, "Complex waves on shielded lossless rectangular dielectric image guide," Electron. Lett., vol. 19, pp. 557-558, Jul. 1983.

[23] M. Mrozowski and J. Mazur, "Matrix theory approach to complex waves," IEEE Trans. Microw. Theory Tech., vol. 40, no. 4, pp. 781-785, Apr. 1992.

[24] F. Elek and G. V. Eleftheriades, "Dispersion analysis of the shielded Sievenpiper structure using multiconductor transmission line theory," IEEE Microw. Wireless Compon. Lett., vol. 14, no. 9, pp. 434-436, Sep. 2004.

[25] M. Beruete, F. Falcone, M. J. Freire, R. Marqués, and J. D. Baena, "Electroinductive waves in chains of complementary metamaterial elements," Appl. Phys. Lett., vol. 88, no. 8, Feb. 2006, Art. ID 083503.

[26] J. Bonache, M. Gil, I. Gil, J. Garcia-García, and F. Martín, "On the electrical characteristics of complementary metamaterial resonators," IEEE Microw. Wireless Compon. Lett., vol. 16, no. 10, pp. 543-545, Oct. 2006

[27] M. Gil, J. Bonache, I. Gil, J. García-García, and F. Martín, "On the transmission properties of left handed microstrip lines implemented by complementary split rings resonators," Int. J. Numer. Model.: Electron. Netw., Devices Fields, vol. 19, pp. 87-103, Mar. 2006.

[28] C. H. Wu, C. H. Wang, and C. H. Chen, "Novel balanced coupled-line bandpass filters with common-mode noise suppression," IEEE Trans. Microw. Theory Tech., vol. 55, no. 2, pp. 287-295, Feb. 2007.

[29] B. L. Teck and Z. Lei, "A differential-mode wideband bandpass filter on microstrip line for UWB applications," IEEE Microw. Wireless Compon. Lett., vol. 19, no. 10, pp. 632-634, Oct. 2009.

[30] J. Shi and Q. Xue, "Novel balanced dual-band bandpass filter using coupled stepped-impedance resonators," IEEE Microw. Wireless Compon. Lett., vol. 20, no. 1, pp. 19-21, Jan. 2010.

[31] J. Shi and Q. Xue, "Dual-band and wide-stopband single-band balanced bandpass filters with high selectivity and common-mode suppression," IEEE Trans. Microw. Theory Tech., vol. 58, no. 8, pp. 2204-2212, Aug. 2010.

[32] J. Shin and Q. Xue, "Balanced bandpass filters using center-loaded half-wavelength resonators," IEEE Trans. Microw. Theory Tech., vol. 58, no. 4, pp. 970-977, Apr. 2010.

[33] C.-H. Wu, C.-H. Wang, and C. H. Chen, "Stopband-extended balanced bandpass filter using coupled stepped-impedance resonators," IEEE Microw. Wireless Compon. Lett., vol. 17, no. 7, pp. 507-509, Jul. 2007.

[34] C.-H. Lee, C.-I. G. Hsu, and C.-C. Hsu, "Balanced dual-band BPF with stub-loaded SIRs for common-mode suppression," IEEE Microw. Wireless Compon. Lett., vol. 20, no. 2, pp. 70-73, Feb. 2010.

[35] J.-S. Hong and M. J. Lancaster, "Lowpass and Bandpass Filters and Coupled Resonator Circuits," in Microstrip Filters for RF/Microwave Applications. New York: Wiley, 2001.

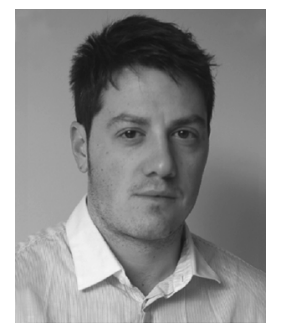

Jordi Naqui (S'11) was born in Granollers, Spain, in 1984. He received the Telecommunication Technical Engineering diploma and Telecommunication Engineering degree from the Universitat Autònoma de Barcelona, Belleterra, Spain, in 2006 and 2010, respectively, where he is currently working toward the Ph.D. degree.

His research subjects are related to innovative passive microwave devices based on metamaterial concepts.

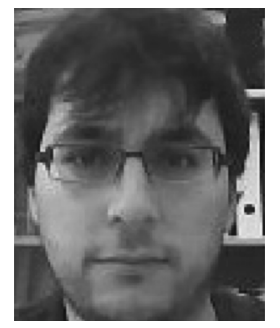

Armando Fernández-Prieto (S'11) was born in Ceuta, Spain, in 1981. He received the Licenciado degree in physics from the Universidad de Sevilla, Seville, Spain, in 2006, where he is currently working toward the Ph.D. degree in electronics and electromagnetism.

His research focuses on printed passive microwave filters, couplers, and metamaterials.

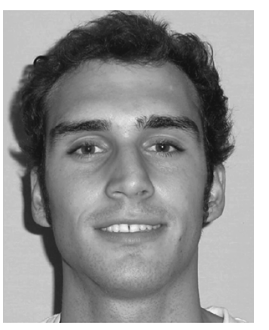

Miguel Durán-Sindreu (S'09-M'11) was born in Barcelona, Spain, in 1985. He received the Telecommunications Engineering diploma, Telecommunications Engineering degree, and $\mathrm{Ph} . \mathrm{D}$. degree from the Universitat Autònoma de Barcelona, Belleterra, Spain, in 2007, 2008 and 2011, respectively.

His research interests are microwave filters, metamaterials, and multiband components.

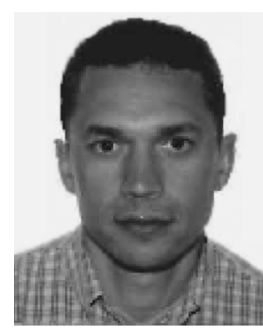

Francisco Mesa (M'93-SM'01) was born in Cádiz, Spain, in April 1965. He received the Licenciado and Ph.D. degrees from the University of Seville, Seville, Spain, in 1989 and 1991, respectively, both in physics.

He is currently a Professor with the Department of Applied Physic 1, University of Seville, Seville, Spain. His research interests focus on electromagnetic propagation/radiation in planar structures.

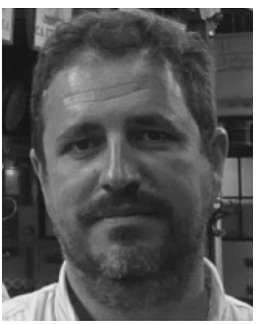

Jesús Martel (M'08) was born in Seville, Spain, in 1966. He received the Licenciado and Ph.D. degrees from the University of Seville, Seville, Spain, in 1989 and 1996, respectively, both in physics.

Since 1992, he has been with the Department of Applied Physics II, University of Seville, Seville, Spain, where, in 2000, he became an Associate Professor. His current research interests are focused on the numerical analysis of planar transmission lines, the modeling of planar microstrip discontinuities, the design of passive microwave circuits, microwave measurements, and artificial media. 


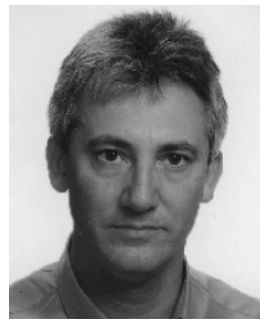

Francisco Medina (M'90-SM'01-F'10) was born in Cádiz, Spain, in November 1960. He received the Licenciado and Ph.D. degrees from the University of Seville, Seville, Spain, in 1983 and 1987 respectively, both in physics.

From 1986 to 1987, he spent the academic year with the Laboratoire de Microondes de l'ENSEEIHT, Toulouse, France, as the recipient of a Spanish Ministry of Science and Education Research Scholarship. From 1985 to 1989, he was an Assistant Professor with the Department of Electronics and Electromagnetism, University of Seville, Seville, Spain, where, since 1990, he has been an Associate Professor of electromagnetism. Since July 2009, he has been a Full Professor of electromagnetism and Head of the Microwaves Group. His research interests include analytical and numerical methods for guiding, resonant, and radiating structures, passive planar circuits, periodic structures, and the influence of anisotropic materials (including microwave ferrites) on such systems. He is also interested in artificial media modeling and extraordinary transmission phenomena. He is on the Editorial Board of the International Journal of RF and Microwave Computer-Aided Engineering.

Dr. Medina is a Fellow of the Massachusetts Institute of Technology (MIT) Electromagnetics Academy. He is a reviewer of the IEEE TRANSACTIONS ON MiCROWAVE THEORY AND TECHNIQUES and of many other journals. He has been a member of the Technical Programme Committees (TPC) of several international and local conferences and has organized a few conferences and workshops.

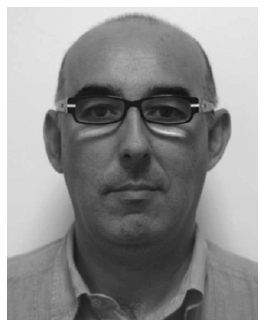

Ferran Martín (M'04-SM'08-F'12) was born in Barakaldo Spain, in 1965. He received the B.S. degree in physics and Ph.D. degree from the Universitat Autònoma de Barcelona, Belleterra, Spain, in 1988 and 1992, respectively.

From 1994 to 2006, he was an Associate Professor in electronics with the Departament d'Enginyeria Electrònica, Universitat Autònoma de Barcelona (UAB), Belleterra, Spain, and since 2007 he has been a Full Professor of electronics. In recent years, he has been involved in different research activities including modelling and simulation of electron devices for high-frequency applications, millimeter-wave and terahertz generation systems, and the application of electromagnetic bandgaps to microwave and millimeter-wave circuits. $\mathrm{He}$ is now very active in the field of metamaterials and their application to the miniaturization and optimization of microwave circuits and antennas. He is the head of the Microwave and Millimeter Wave Engineering Group (GEMMA Group) at UAB, and director of CIMITEC, a research Center on Metamaterials supported by TECNIO (Generalitat de Catalunya). He has authored and co-authored over 350 technical conference, letter and journal papers and he is coauthor of the monograph on metamaterials, entitled Metamaterials with Negative Parameters: Theory, Design and Microwave Applications (Wiley, 2008). He has filed several patents on metamaterials and has headed several Development Contracts.

Dr. Martín received the 2006 Duran Farell Prize for Technological Research, he holds the Parc de Recerca UAB - Santander Technology Transfer Chair, and he was the recipient of an ICREA ACADEMIA Award. He has organized several international events related to metamaterials, including Workshops at the IEEE Microwave Theory and Techniques Society (IEEE MTT-S) International Microwave Symposium (years 2005 and 2007) and European Microwave Conference (2009). He has acted as a guest editor for three Special Issues on metamaterials in three international journals. 